\title{
Degree-constrained graph partition
}

\author{
Thinh D. Nguyen
}

\begin{abstract}
In this paper, we consider the task of partitioning a given graph intwo two non-empty subgraphs such that one of them has no vertex of degree less than 2, the other has no vertex of degree less than 3. We show that this problem is a difficult one.
\end{abstract}

Keywords. degree, graph, partition

\section{Definitions and notations}

First, some notations are used throughout the paper is given. Given a graph $G=(V, E)$, a subset $V_{1} \subset V$ induce an induced subgraph $G\left[V_{1}\right]$ of $G$. The minimum degree of the vertices of $G$ is denoted by $\delta(G)$.

Second, we formally define our problem now.

Definition 1. (Problem Degree-constrained Graph Partition)

Input: A graph $G=(V, E)$

Output: Decide whether there exists a partition of $G$, namely $\left(V_{1}, V_{2}\right)$, where $V_{1} \cup V_{2}=V$ such that $\delta\left(G\left[V_{1}\right]\right) \geq 2$ and $\delta\left(G\left[V_{2}\right]\right) \geq 3$.

Now, we state the main claim of this paper.

\section{Claim 2. 3 -Sat $\leq_{p}$ Degree-Constrained Graph Partition}

The construction in our reduction is rather involed which will be descrbed in details in the following section.

\section{Description of the construction}

Proof. For convenience of description, we consider the problem as a coloring problem where vertices in $V_{1}$ are colored red and vertices in $V_{2}$ are colored blue.

The following is a reduction from 3SAT.

For any instance of 3SAT with $n$ variables (assume $n \geq 4$ without loss of generality), construct a complete graph of $2 n$ vertices $V=\left\{v_{1}^{0}, v_{1}^{1}, \ldots, v_{n}^{0}, v_{n}^{1}\right\}$ where $v_{i}^{0}, v_{i}^{1}$ respectively represent the positive and negative literals of the $i$ th variable. We also add some additional structures. Note that only named vertices can be in more than one structure. 
1. For each $i$, add the following structure (note we emit the edges among named vertices and the same below):

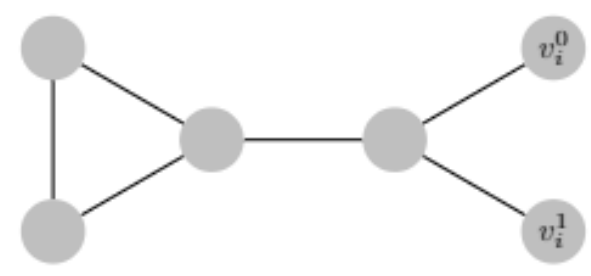

This structure ensures all the unnamed vertices are colored red, and at least one of $v_{i}^{0}$ and $v_{i}^{1}$ must be colored red.

2. For each clause $l_{1} \vee l_{2} \vee l_{3}$, say their corresponding vertices are $u, v, w \in$ $V$, add the following structure:

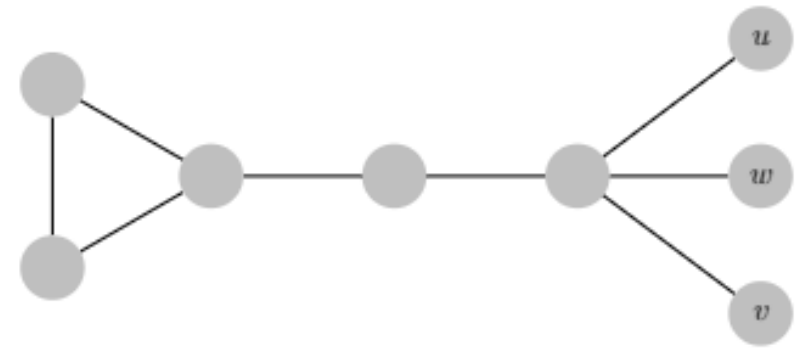

This structure ensures all the unnamed vertices are colored red, and at least one of $u, v, w$ must be colored red.

3. For each $i$, for each pair of vertices $\{u, v\} \subseteq V$, add the following structure:

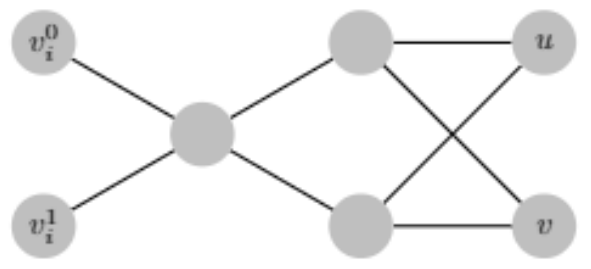

If both $v_{i}^{0}$ and $v_{i}^{1}$ are colored red, then all the unnamed vertices must be colored red, and at least one of $u$ and $v$ are colored red. Note this holds for all pairs $\{u, v\}$, so if both $v_{i}^{0}$ and $v_{i}^{1}$ are colored red, there are at most one vertex colored blue, which is impossible. As a result, 
this structure ensures at least one of $v_{i}^{0}$ and $v_{i}^{1}$ is colored blue. Combined with the first type of structures, it is ensured that $v_{i}^{0}$ and $v_{i}^{1}$ are colored different colors.

Now if there is a proper coloring, exactly one of $v_{i}^{0}$ and $v_{i}^{1}$ is colored red. If it is $v_{i}^{0}$, assign the $i$ th variable 1 , otherwise 0 , then the assignment is a valid assignment for the $3 \mathrm{SAT}$ instance.

On the other hand, if there is a valid assignment for the 3SAT instance, color $v_{i}^{0}$ red and $v_{i}^{1}$ blue if the $i$ th variable is assigned 1 , otherwise color $v_{i}^{0}$ blue and $v_{i}^{1}$ red. Note the subgraph induced by $\left\{v_{1}^{0}, v_{1}^{1}, \ldots, v_{n}^{0}, v_{n}^{1}\right\}$ is a complete graph, this is a legal coloring. All unnamed vertices in the first two kinds of structures are colored red, and for a structure of the third type, if both $u$ and $v$ are colored blue, color all the three unnamed vertices blue, otherwise color them red. This is a proper coloring. 